\title{
NOVEL INVARIANTS FOR ALMOST GEODESIC MAPPINGS OF THE THIRD TYPE
}

\author{
DUŠAN J. SIMJANOVIĆ AND NENAD O. VESIĆ
}

Received 13 October, 2020

\begin{abstract}
Two kinds of invariance for geometrical objects under transformations are involved in this paper. Based on these kinds, we obtained new invariants for almost geodesic mappings of the third type of a non-symmetric affine connection space in this paper. Our results are presented in two sections. In the Section 3, we obtained the invariants for the equitorsion almost geodesic mappings which do not have the property of reciprocity.
\end{abstract}

2010 Mathematics Subject Classification: 53B05; 15A72; 53A55; 53A25

Keywords: mapping, invariance, almost geodesics

\section{INTRODUCTION}

Many authors have obtained invariants for different mappings between symmetric and non-symmetric affine connection spaces. Some of them are J. Mikeš and his research group [1-3, 5, 15], N. S. Sinyukov [14], M. S. Stanković [13, 18, 19, 21], M. Lj. Zlatanović [22,23], M. Z. Petrović [12,13] and many others.

\subsection{Affine connection spaces}

An $N$-dimensional manifold equipped with non-symmetric affine connection $\nabla$ (see Eisenhart [4]) is the non-symmetric affine connection space $\mathbb{G A}_{N}$ (in Eisenhart's sense).

Affine connection coefficients of the space $\mathbb{G A}_{N}$ are $L_{j k}^{i}, L_{j k}^{i} \not \equiv L_{k j}^{i}$ for at least one pair $(j, k) \in\{1, \ldots, N\} \times\{1, \ldots, N\}$. For this reason, symmetric and anti-symmetric parts of affine connection coefficients $L_{j k}^{i}$ are

$$
L_{\underline{j k}}^{i}=\frac{1}{2}\left(L_{j k}^{i}+L_{k j}^{i}\right) \quad \text { and } \quad L_{j k}^{i}=\frac{1}{2}\left(L_{j k}^{i}-L_{k j}^{i}\right) .
$$

${ }^{*}$ This paper is financially supported by Serbian Ministry of Education, Science and Technological Development through the Mathematical Institute of Serbian Academy of Sciences and Arts. 
The $N$-dimensional manifold equipped with torsion-free affine connection $\stackrel{0}{\nabla}$ whose coefficients are $L_{j k}^{i}$ is associated space $\mathbb{A}_{N}\left(\right.$ of space $\left.\mathbb{G A}_{N}\right)$ [20,21].

Based on affine connection $\stackrel{0}{\nabla}$, one kind of covariant derivative exists $[2,3,5,14,15]$

$$
a_{j \mid k}^{i}=a_{j, k}^{i}+L_{\underline{\alpha k}}^{i} a_{j}^{\alpha}-L_{\underline{j k}}^{\alpha} a_{\alpha}^{i},
$$

for partial derivative $\partial / \partial x^{k}$ denoted by comma.

In this case, one Ricci identity $a_{j \mid m n}^{i}-a_{j \mid n m}^{i}=a_{j}^{\alpha} R_{\alpha m n}^{i}-a_{\alpha}^{i} R_{j m n}^{\alpha}$ exists. Curvature tensor $R_{j m n}^{i}$ of associated space $\mathbb{A}_{N}$ is

$$
R_{j m n}^{i}=L_{\underline{j m, n}}^{i}-L_{\underline{j n, m}}^{i}+L_{\underline{j m}}^{\alpha} L_{\underline{\alpha n}}^{i}-L_{\underline{j n}}^{\alpha} L_{\underline{\alpha m}}^{i} .
$$

Geometrical object

$$
R_{i j}=R_{i j \alpha}^{\alpha}
$$

is the Ricci tensor.

Based on non-symmetric affine connection $\nabla, \mathrm{S}$. M. Minčić defined four kinds of covariant derivatives $[7,8]$

$$
\begin{array}{ll}
a_{j \mid k}^{i}=a_{j, k}^{i}+L_{\alpha k}^{i} a_{j}^{\alpha}-L_{j k}^{\alpha} a_{\alpha}^{i}, & a_{j \mid k}^{i}=a_{j, k}^{i}+L_{k \alpha}^{i} a_{j}^{\alpha}-L_{k j}^{\alpha} a_{\alpha}^{i} \\
a_{j \mid k}^{i}=a_{j, k}^{i}+L_{\alpha k}^{i} a_{j}^{\alpha}-L_{k j}^{\alpha} a_{\alpha}^{i}, & a_{j \mid k}^{i}=a_{j, k}^{i}+L_{k \alpha}^{i} a_{j}^{\alpha}-L_{j k}^{\alpha} a_{\alpha}^{i}
\end{array}
$$

\section{Explanation of covariant derivatives [10]:}

Two kinds of parallel displacement of vectors in $\mathbb{G A}_{N}$ exist. For a vector field $v^{i}=v^{i}(t)$, defined along a curve $C: x^{i}=x^{i}(t), v^{i}$ is a field of $k$-th, $k=1,2$, parallel transport if for the differential one has

$$
\underset{k}{d v^{i}}=-\left(L_{\underline{\alpha \beta}}^{i}-(-1)^{k} L_{\alpha \beta}^{i}\right) v^{\alpha} d x^{\beta} .
$$

After considering a surface element at the tangent space determined by two infinitesimal vectors whose origin is the point $P\left(x^{i}\right)$. The ends of these vectors are $Q\left(x^{i}+d x^{i}\right)$ and $R\left(x^{i}+\delta x^{i}\right)$.

If we make the parallel displacement of the first kind of the vector $d x^{i}$ along $\delta x^{i}$ and $\delta x^{i}$ along $d x^{i}$, we obtain different points $S$ and $T$ for the ends. The coordinates of these points are

$$
{\underset{T}{x}}_{T}^{i}-x_{S}^{i}=\underset{1}{d}\left(\delta x^{i}\right)-\underset{1}{\delta}\left(d x^{i}\right)=\left(L_{\alpha \beta}^{i}-L_{\beta \alpha}^{i}\right) d x^{\alpha} \delta x^{\beta}=2 L_{\alpha \beta}^{i} d x^{\alpha} \delta x^{\beta} .
$$

From the last equation, we can conclude that the points $S$ and $T$ are different if $L_{j k}^{i} \neq 0$. 
Based on Ricci identities $a_{\substack{j|m| n \\ p}}^{i}-a_{\substack{j|n| m \\ r}}^{i}, p, q, r, s \in\{0, \ldots, 4\}, \underset{0}{a_{j \mid k}^{i}}=a_{j \mid k}^{i}$, the family of curvature tensors for the space $\mathbb{G A}_{N}$ is obtained [6-8]

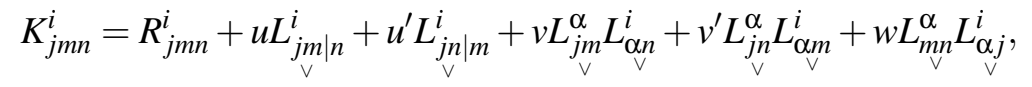

for curvature tensor $R_{j m n}^{i}$ of associated space $\mathbb{A}_{N}$ and scalars $u, u^{\prime}, v, v^{\prime}, w$.

Family of Ricci-curvatures $K_{i j}=K_{i j \alpha}^{\alpha}$ of space $\mathbb{G A}_{N}$ is

$$
K_{i j}=R_{i j}+u L_{\vee j \mid \alpha}^{\alpha}+u^{\prime} L_{i \alpha \mid j}^{\alpha}+v L_{\vee j}^{\alpha} L_{\underset{\vee}{\alpha \beta}}^{\beta}-\left(v^{\prime}+w\right) L_{\vee}^{\alpha} L_{\vee}^{\beta} L_{\vee}^{\beta} .
$$

\subsection{Geodesic and almost geodesic lines and almost geodesic mappings}

Curve $\ell=\ell(t)$ in associated space $\mathbb{A}_{N}$ whose tangential vector $\lambda=\frac{d \ell}{d t}$ satisfies the system of differential equations $[5,14]$

$$
\frac{d \lambda^{i}}{d t}+L_{\underline{\alpha \beta}}^{i} \lambda^{\alpha} \lambda^{\beta}=\zeta \lambda^{i}
$$

for scalar $\zeta$, is the geodesic line of space $\mathbb{A}_{N}$.

Definition 1. $[2,3,5,14,15]$ A curve $\tilde{\bar{\ell}}=\tilde{\bar{\ell}}(t)$ in associated space $\overline{\mathbb{A}}_{N}$ whose tangential vector $\tilde{\bar{\lambda}}=\frac{d \tilde{\bar{\lambda}}}{d t}$ satisfies the system of equations

$$
\tilde{\bar{\lambda}}_{2}^{i}=\bar{a}(t) \tilde{\lambda}^{i}+\bar{b}(t) \tilde{\bar{\lambda}}_{1}^{i}, \quad \tilde{\bar{\lambda}}_{1}^{i}=\tilde{\lambda}_{\| \alpha}^{i} \tilde{\lambda}^{\alpha}, \quad \tilde{\bar{\lambda}}_{2}^{i}=\tilde{\lambda}_{(1) \| \alpha}^{i} \tilde{\lambda}^{\alpha},
$$

for functions $\bar{a}(t), \bar{b}(t)$ and covariant derivative with respect to the affine connection of the space $\overline{\mathbb{A}}_{N}$ denoted by $\|$, is almost geodesic line of space $\overline{\mathbb{A}}_{N}$.

Mapping $f: \mathbb{A}_{N} \rightarrow \overline{\mathbb{A}}_{N}$ is an almost geodesic mapping if any geodesic line of $\mathbb{A}_{N}$ transforms to an almost geodesic line in $\overline{\mathbb{A}}_{N}$ (see $\left.[2,3,5,14,15]\right)$.

Sinjukov proved [14] that three types of almost geodesic mappings of a space $\mathbb{A}_{N}$ exist. These types are $\pi_{1}, \pi_{2}, \pi_{3}$.

It was proved [14] that the inverse mapping of almost geodesic one $f: \mathbb{A}_{N} \rightarrow \overline{\mathbb{A}}_{N}$ of the type $\pi_{3}$ is almost geodesic mapping of the type $\pi_{3}$. Almost geodesic mappings of the third type whose inverse transformations are almost geodesic mappings of the third type have the property of reciprocity (see [14], page 191).

M. S. Stanković [16-18] generalized the Sinyukov's concept of almost geodesic mappings. A curve $\tilde{\bar{\ell}}=\tilde{\bar{\ell}}(t)$ whose tangential vector $\tilde{\bar{\lambda}}=\frac{d \tilde{\bar{\ell}}}{d t}$ is solution of the system $[12,13,16-19,21]$ 


$$
\tilde{\bar{\lambda}}_{k}^{i}(2)=\bar{a}(t) \tilde{\lambda}^{i}+\underset{k}{\bar{b}}(t) \underset{k}{\tilde{\bar{\lambda}}^{i}(1)}, \quad \tilde{\bar{\lambda}}_{k}^{i}(1)=\tilde{\lambda}_{k}^{i} \tilde{\lambda}^{\alpha}, \quad \tilde{\bar{\lambda}}_{k}^{i}(2)=\underset{k}{(1) \| \alpha} \tilde{\bar{\lambda}}_{k}^{i} \tilde{\lambda}^{\alpha},
$$

$k \in\{1,2\}$, of differential equations is an almost geodesic line of the $k$-th kind of space $\mathbb{G}_{N}$.

Mapping $f: \mathbb{G A}_{N} \rightarrow \mathbb{G}_{N}$ that any geodesic line of space $\mathbb{G A}_{N}$ transforms to an almost geodesic line of the $k$-th type, $k=1,2$, of space $\mathbb{G}_{\mathbb{A}}$ is almost geodesic mapping of space $\mathbb{G A}_{N}$.

A mapping $f: \mathbb{G A}_{N} \rightarrow \mathbb{G}_{\mathbb{A}_{N}}$ is equitorsion [9] if anti-symmetric parts $L_{j k}^{i}$ and $\bar{L}_{j k}^{i}$ of affine connection coefficients $L_{j k}^{i}$ and $\bar{L}_{j k}^{i} \stackrel{f}{\leftarrow} L_{j k}^{i}$ of spaces $\mathbb{G A}_{N}$ and $\mathbb{G}_{N}$ are equal. In this paper, we will stay focused on equitorsion almost geodesic mappings of the third type and first kind.

Motivated by the Sinyukov's research [14], M. S. Stanković [16-18] determined two types of almost geodesic lines. Any of three types of almost geodesic mappings can be divided into two subtypes. These subtypes of almost geodesic mappings are $\pi_{r}, \pi_{2}, r=1,2,3$.

An almost geodesic mapping of a subtype $\underset{k}{\pi_{3}}, k=1,2$, has the property of reciprocity if its inverse mapping is almost geodesic mapping of the same subtype. Unlike almost geodesic mappings of symmetric affine connection spaces, almost geodesic mappings of a non-symmetric affine connection space of the third type do not have the property of reciprocity in general.

We will obtain invariants for almost geodesic mappings of the third type of space $\mathbb{G A}_{N}$ which have or do not have the property of reciprocity below.

\subsection{Two kinds of invariants for transformations}

Invariants for mappings between symmetric and non-symmetric affine connection spaces are such geometric objects whose values and forms do not change under the acting of the corresponding mapping. If almost geodesic mappings $f: \mathbb{G A}_{N} \rightarrow \mathbb{G}^{\mathbb{A}_{N}}$ or $f: \mathbb{A}_{N} \rightarrow \overline{\mathbb{A}}_{N}$ do not have the property of reciprocity, invariants for these mappings of the common values and forms cannot be obtained. For this reason, to obtain the invariants for almost geodesic mappings based on changes of the curvature tensors under almost geodesic mappings, authors assume that these mappings have the property of reciprocity.

Two kinds of invariance are important in physics (taken from the textbook Đ. Mušicki, B. Milić, Mathematical Foundations of Theoretical Physics With a Collection of Solved Problems [11], page 103):

- The invariant of a mapping $f$ is an object whose value stays saved but form changes under transformation of coordinates under this mapping. 
- The total invariant of a mapping $f$ is an object whose value and form stay saved under the transformation of coordinates under this mapping.

Invariants for geometric mappings that have been obtained so far are analogies to the total invariants from physics. To debilitate this condition for invariance of geometrical objects, we give the following definition.

Definition 2. Let $f: \mathbb{G A}_{N} \rightarrow \mathbb{G} \overline{\mathbb{A}}_{N}$ be a mapping and let $U_{j_{1} \ldots j_{q}}^{i_{1} \ldots i_{p}}$ be a geometrical object of the type $(p, q)$.

- If the transformation $f$ preserves the value of the object $U_{j_{1} \ldots j_{q}}^{i_{1} \ldots i_{p}}$ but changes its form to $\bar{V}_{j_{1} \ldots j_{q}}^{i_{1} \ldots i_{p}}$, then the invariance for geometrical object $U_{j_{1} \ldots j_{q}}^{i_{1} \ldots i_{p}}$ under transformation $f$ is valued.

- If the transformation $f$ preserves both the value and the form of the geometrical object $U_{j_{1} \ldots j_{q}}^{i_{1} \ldots i_{p}}$, then the invariance for the geometrical object $U_{j_{1} \ldots j_{q}}^{i_{1} \ldots i_{p}}$ under transformation $f$ is total.

Example 1. An invariant for geodesic mapping $f: \mathbb{A}_{N} \rightarrow \overline{\mathbb{A}}_{N}$ is the Weyl projective tensor

$$
W_{j m n}^{i}=R_{j m n}^{i}+\frac{1}{N+1} \delta_{j}^{i} R_{[m n]}+\frac{N}{N^{2}-1} \delta_{[m}^{i} R_{j n]}+\frac{1}{N^{2}-1} \delta_{[m}^{i} R_{n] j},
$$

for $R_{[m n]}=R_{m n}-R_{n m}, \delta_{[m}^{i} R_{j n]}=\delta_{m}^{i} R_{j n}-\delta_{n}^{i} R_{j m}, \delta_{[m}^{i} R_{n] j}=\delta_{m}^{i} R_{n j}-\delta_{n}^{i} R_{m} j$.

The geodesic mapping $f$ transforms Weyl projective tensor $W_{j m n}^{i}$ to

$$
\bar{W}_{j m n}^{i}=\bar{R}_{j m n}^{i}+\frac{1}{N+1} \delta_{j}^{i} \bar{R}_{[m n]}+\frac{N}{N^{2}-1} \delta_{[m}^{i} \bar{R}_{j n]}+\frac{1}{N^{2}-1} \delta_{[m}^{i} \bar{R}_{n] j} .
$$

It holds $W_{j m n}^{i}=\bar{W}_{j m n}^{i}$ and the forms of the tensors $W_{j m n}^{i}$ and $\bar{W}_{j m n}^{i}$ are equal. For this reason, the invariant $W_{j m n}^{i}$ is total.

If $R_{i j} \not \equiv R_{j i}$ and $\bar{R}_{i j} \equiv \bar{R}_{j i}$ as it is the case in [23], the form of invariant $W_{j m n}^{i}$ stays same but the invariant $\bar{W}_{j m n}^{i}$ changes to

$$
\overline{\bar{W}}_{j m n}^{i}=\bar{R}_{j m n}^{i}+\frac{1}{N-1}\left(\delta_{m}^{i} R_{j n}-\delta_{n}^{i} R_{j m}\right) .
$$

The equality $W_{j m n}^{i}=\overline{\bar{W}}_{j m n}^{i}$ holds (the values of geometrical objects $W_{j m n}^{i}$ and $\overline{\bar{W}}_{j m n}^{i}$ are equal) but their forms are different. For this reason, the invariant $W_{j m n}^{i}$ is valued.

The transformations which are the main subject of research in this paper are almost geodesic mappings of the third type.

\subsection{Motivation}

The transformation rules for affine connection coefficients under the third type almost geodesic mappings of symmetric and non-symmetric affine connection spaces are 


$$
\begin{aligned}
& L_{j k}^{i}=L_{\underline{j k}}^{i}+\psi_{j} \delta_{k}^{i}+\psi_{k} \delta_{j}^{i}+\sigma_{j} k \varphi^{i}, \\
& \bar{L}_{j k}^{i}=L_{j k}^{i}+\psi_{j} \delta_{k}^{i}+\psi_{k} \delta_{j}^{i}+\sigma_{j} k \varphi^{i}+\xi_{j k}^{i},
\end{aligned}
$$

for the 1 -form $\psi_{i}$, the contravariant vector $\varphi^{i}$ and the tensors $\sigma_{j k}$ and $\xi_{j k}^{i}$ symmetric and anti-symmetric in the covariant indices $j$ and $k$, respectively.

To generalize the Weyl projective tensor by an invariant for a third type almost geodesic mapping of symmetric affine connection space $\mathbb{A}_{N}$, N. S. Sinyukov involved (see [14], page 193) geometrical object $q_{i}$ so that $q_{\alpha} \varphi^{\alpha}=e, e= \pm 1$. After some computations, Sinyukov generalized the Thomas projective parameter and the Weyl projective tensor as invariants for almost geodesic mapping $f$.

M. S. Stanković (see [18]) continued Sinyukov's research about invariants for almost geodesic mappings of the third type. Motivated by the results presented in [18], N. O. Vesić, Lj. S. Velimirović and M. S. Stanković [21] obtained the family of invariants for the equitorsion third type almost geodesic mappings of a non-symmetric affine connection space. We will generalize this result below.

N. S. Sinyukov [14] generalized the Weyl projective tensor as invariant for the third type almost geodesic mappings of a symmetric affine connection space. M. S. Stanković [18] obtained one generalization of the Weyl projective tensor as invariant for the third type almost geodesic mapping of a non-symmetric affine connection space based on the change of the curvature tensor of the corresponding associated space. N. O. Vesić, Lj. S. Velimirović, M. S. Stanković [21] obtained one family of invariants for the third type almost geodesic mappings of a non-symmetric affine connection space which generalizes the Weyl projective tensor.

In [20], two invariants for mappings of an associated space analogue to the Weyl projective tensor (called the invariants of the Weyl type) are obtained. That motivated us to obtain invariants for almost geodesic mappings of the third type of a non-symmetric affine connection space.

The formulae of invariants for mappings between symmetric and non-symmetric affine connection spaces are obtained in [20]. We will use these formulae to meet the main goals of this paper. These goals are:

(1) To obtain invariants for equitorsion almost geodesic mappings of a nonsymmetric affine connection space.

(2) To obtain necessary and sufficient conditions for these invariants to be total.

\section{REVIEW OF BASIC INVARIANTS}

In [20], invariants for mappings $f: \mathbb{G A}_{N} \rightarrow \mathbb{G}_{N}$ are obtained. If deformation tensor $P_{j k}^{i}=\bar{L}_{j k}^{i}-L_{j k}^{i}$ of mapping $f$ is

$$
P_{j k}^{i}=\bar{L}_{j k}^{i}-L_{j k}^{i}=\bar{\omega}_{j k}^{i}-\omega_{j k}^{i}+\bar{\tau}_{j k}^{i}-\tau_{j k}^{i},
$$


for $\omega_{j k}^{i}=\omega_{k j}^{i}, \bar{\omega}_{j k}^{i}=\bar{\omega}_{k j}^{i}, \tau_{j k}^{i}=-\tau_{k j}^{i}, \bar{\tau}_{j k}^{i}=-\bar{\tau}_{k j}^{i}$, basic associated invariants of Thomas and Weyl type for mapping $f$ are

$$
\begin{aligned}
\widetilde{\mathcal{T}}^{i}{ }_{j k} & =L_{j k}^{i}-\omega_{j k}^{i}, \\
\widetilde{\mathcal{W}}_{j m n}^{i} & =R_{j m n}^{i}-\omega_{j m \mid n}^{i}+\omega_{j n \mid m}^{i}+\omega_{j m}^{\alpha} \omega_{\alpha n}^{i}-\omega_{j n}^{\alpha} \omega_{\alpha m}^{i} .
\end{aligned}
$$

Remark 1. The geometrical object $\omega_{j k}^{i}$ depends of a mapping $f$. If the mapping $f: \mathbb{G A}_{N} \rightarrow \mathbb{G}_{\mathbb{A}_{N}}$ is geodesic, this object is $\omega_{j k}^{i}=L_{j \alpha}^{\alpha} \delta_{k}^{i}+L_{k \alpha}^{\alpha} \delta_{j}^{i}$. If the mapping $f: \mathbb{G A}_{N} \rightarrow \mathbb{G R}_{N}$ is almost geodesic of the third type, the object $\omega_{j k}^{i}$ will be different as we will se below.

To simplify these formulae, the next geometrical object is used [20]

In case of

$$
L_{\underline{j m} \mid n}^{i}=L_{\underline{j m, n}}^{i}+L_{\underline{\alpha n}}^{i} L_{\underline{j m}}^{\alpha}-L_{\underline{j n}}^{\alpha} L_{\underline{\alpha m}}^{i}-L_{\underline{m n}}^{\alpha} L_{\underline{j \alpha}}^{i} .
$$

$$
\omega_{j k}^{i}=\delta_{k}^{i} \rho_{j}+\delta_{j}^{i} \rho_{k}+\sigma_{j k}^{i}
$$

for $\sigma_{j k}^{i}=\sigma_{k j}^{i}$, the invariants for the mapping $f$ given by the equations $(2.2,2.3)$ transform to

$$
\begin{aligned}
\widetilde{T}_{j k}^{i}= & L_{j k}^{i}-\sigma_{j k}^{i}-\frac{1}{N+1}\left(\left(L_{\underline{j \alpha}}^{\alpha}-\sigma_{j \alpha}^{\alpha}\right) \delta_{k}^{i}+\left(L_{k \alpha}^{\alpha}-\sigma_{k \alpha}^{\alpha}\right) \delta_{j}^{i}\right), \\
\widetilde{W}_{j m n}^{i}= & R_{j m n}^{i}-\delta_{[m}^{i} \rho_{j \mid n]}-\delta_{j}^{i} \rho_{[m \mid n]}-\sigma_{j[m \mid n]}^{i}-\delta_{[m}^{i} \rho_{j} \rho_{n]} \\
& +\delta_{[m}^{i} \rho_{\alpha} \sigma_{j n]}^{\alpha}+\sigma_{j[m}^{\alpha} \sigma_{\alpha n]}^{i},
\end{aligned}
$$

The derived invariant of Weyl type for mapping $f$ is [20]

$$
\begin{aligned}
\widetilde{W}_{j m n}^{i}= & R_{j m n}^{i}+\frac{1}{N+1} \delta_{j}^{i}\left(R_{[m n]}+\sigma_{\alpha[m \mid n]}^{\alpha}\right)+\frac{N}{N^{2}-1} \delta_{[m}^{i} R_{j n]}+\frac{1}{N^{2}-1} \delta_{[m}^{i} R_{n] j} \\
& -\sigma_{j m \mid n}^{i}+\sigma_{j n \mid m}^{i}+\sigma_{j m}^{\alpha} \sigma_{\alpha n}^{i}-\sigma_{j n}^{\alpha} \sigma_{\alpha m}^{i} \\
& -\frac{1}{N^{2}-1} \delta_{m}^{i}\left(\sigma_{\alpha[j \mid n]}^{\alpha}+(N+1)\left(\sigma_{j n \mid \alpha}^{\alpha}-\sigma_{j \alpha \mid n}^{\alpha}-\sigma_{j n}^{\alpha} \sigma_{\alpha \beta}^{\beta}+\sigma_{j \beta}^{\alpha} \sigma_{n \alpha}^{\beta}\right)\right) \\
& +\frac{1}{N^{2}-1} \delta_{n}^{i}\left(\sigma_{\alpha[j \mid m]}^{\alpha}+(N+1)\left(\sigma_{j m \mid \alpha}^{\alpha}-\sigma_{j \alpha \mid m}^{\alpha}-\sigma_{j m}^{\alpha} \sigma_{\alpha \beta}^{\beta}+\sigma_{j \beta}^{\alpha} \sigma_{m \alpha}^{\beta}\right)\right) .
\end{aligned}
$$

The basic invariant for equitorsion mapping $f: \mathbb{G A}_{N} \rightarrow \mathbb{G}_{N}$ obtained from the transformation of anti-symmetric part $L_{j k}^{i}$ of affine connection coefficient $L_{j k}^{i}$ is

$$
\hat{\mathcal{T}}^{i}{ }_{j k}=L_{j k}^{i} .
$$

Let be $\omega_{(1) . j k}^{i}=L_{\underline{j k}}^{i}, \bar{\omega}_{(1) . j k}^{i}=\bar{L}_{\underline{j k}}^{i}, \omega_{(2) . j k}^{i}=\omega_{j k}^{i}, \bar{\omega}_{(2) . j k}^{i}=\bar{\omega}_{j k}^{i}$. Based on equalities $\hat{\overline{\mathcal{T}}}_{j m \| n}^{i}-\hat{\mathcal{T}}_{j m \mid n}^{i}=P_{\underline{\alpha n}}^{i} \hat{\mathcal{T}}_{j m}^{\alpha}-P_{\underline{j n}}^{\alpha} \hat{\mathcal{T}}_{\alpha n}^{i}-P_{\underline{m n}}^{\alpha} \hat{\mathcal{T}}_{j \alpha}^{i}, P_{\underline{j k}}^{i}=\bar{\omega}_{(1) . j k}^{i}-\omega_{(1) . j k}^{i}, P_{j k}^{i}=$ 
$\bar{\omega}_{(2) . j k}^{i}-\omega_{(2) . j k}^{i}$, we obtain [20]

$$
\overline{\boldsymbol{\theta}}_{(p) . j m n}^{i}=\boldsymbol{\theta}_{(p) . j m n}^{i},
$$

where $p=\left(p_{1}, p_{2}, p_{3}\right), p_{1}, p_{2}, p_{3} \in\{1,2\}$, and

$$
\theta_{(p) . j m n}^{i}=L_{j m \mid n}^{i}-\tau_{j m \mid n}^{i}-\omega_{\left(p_{1}\right) . \alpha n}^{i} \hat{\mathcal{T}}_{j m}^{\alpha}+\omega_{\left(p_{2}\right) . j n}^{\alpha} \hat{\mathcal{T}}_{\alpha m}^{i}+\omega_{\left(p_{3}\right) . m n}^{\alpha} \hat{\mathcal{T}}_{j \alpha}^{i} .
$$

The family of invariants of for equitorsion mapping $f$ obtained based on the transformation of family $K_{j m n}^{i}$ of curvature tensors for space $\mathbb{G A}_{N}$ is [20]

$$
W_{\left(p^{1}\right) \cdot\left(p^{2}\right) \cdot j m n}^{i}=\widetilde{\mathcal{W}}_{j m n}^{i}+u \theta_{\left(p^{1}\right) \cdot j m n}^{i}+u^{\prime} \theta_{\left(p^{2}\right) \cdot j m n}^{i},
$$

for $p^{1}=\left(p_{1}^{1}, p_{2}^{1}, p_{3}^{1}\right), p^{2}=\left(p_{1}^{2}, p_{2}^{2}, p_{3}^{2}\right), p_{j}^{i} \in\{1,2\}$ and the corresponding invariants $\theta_{\left(p^{1}\right) . j m n}^{i}, \theta_{\left(p^{2}\right) . j m n}^{i}$, given by (2.8).

\section{INVARIANTS FOR EQUITORSION ALMOST GEODESIC MAPPINGS}

Let $f: \mathbb{G A}_{N} \rightarrow \mathbb{G}_{N}$ be an equitorsion almost geodesic mapping of the third type. Its basic equations are [18]

$$
\left\{\begin{aligned}
& \bar{L}_{j k}^{i}=L_{j k}^{i}+\psi_{j} \delta_{k}^{i}+\psi_{k} \delta_{j}^{i}+2 \sigma_{j k} \varphi^{i} \\
& \varphi_{\mid j}^{i}=v_{1} \varphi^{i}+\mu \delta_{j}^{i} \\
& 1
\end{aligned}\right.
$$

Let us rewrite the first of last basic equations as

$$
\bar{L}_{\underline{j k}}^{i}=L_{\underline{j k}}^{i}+\psi_{j} \delta_{k}^{i}+\psi_{k} \delta_{j}^{i}+D_{j k}^{i},
$$

for tensor $D_{j k}^{i}, D_{j k}^{i}=D_{k j}^{i}=2 \sigma_{j k} \varphi^{i}$. In case of inverse mapping $f^{-1}: \mathbb{G}_{N} \rightarrow \mathbb{G A}_{N}$, tensor $\bar{D}_{j k}^{i}, \bar{D}_{j k}^{i}=\bar{D}_{k j}^{i}, \bar{D}_{j k}^{i}=-D_{j k}^{i}$, exists, so that

$$
L_{\underline{j k}}^{i}=\bar{L}_{\underline{j k}}^{i}-\psi_{j} \delta_{k}^{i}-\psi_{k} \delta_{j}^{i}-D_{j k}^{i}=\bar{L}_{\underline{j k}}^{i}-\psi_{j} \delta_{k}^{i}-\psi_{k} \delta_{j}^{i}+\bar{D}_{j k}^{i} .
$$

Therefore, the equation (3.1') transforms to

$$
\bar{L}_{j k}^{i}=L_{\underline{j k}}^{i}+\psi_{j} \delta_{k}^{i}+\psi_{k} \delta_{j}^{i}-\frac{1}{2}\left(\bar{D}_{j k}^{i}-D_{j k}^{i}\right) .
$$

After contracting the last equation by $i$ and $k$, we obtain

$$
\psi_{j}=\frac{1}{N+1}\left(\bar{L}_{\underline{j \alpha}}^{\alpha}+\frac{1}{2} \bar{D}_{j \alpha}^{\alpha}\right)-\frac{1}{N+1}\left(L_{j \alpha}^{\alpha}+\frac{1}{2} D_{j \alpha}^{\alpha}\right) .
$$

If we substitute the expression (3.3) in (3.1") and use expression $D_{j k}^{i}=2 \sigma_{j k} \varphi^{i}$, we will obtain

$$
\omega_{j k}^{i}=\frac{1}{N+1} \delta_{k}^{i}\left(L_{\underline{j \alpha}}^{\alpha}+\sigma_{j \alpha} \varphi^{\alpha}\right)+\frac{1}{N+1} \delta_{j}^{i}\left(L_{k \alpha}^{\alpha}+\sigma_{k \alpha} \varphi^{\alpha}\right)-\sigma_{j k} \varphi^{i} .
$$


The second of basic equations (3.1) is equivalent to

$$
\varphi_{\mid j}^{i}=-L_{\alpha j}^{i} \varphi^{\alpha}+v_{1} \varphi^{i}+\mu \delta_{j}^{i}
$$

After comparing the equations (2.5) and (3.5), we read

$$
\rho_{j}=\frac{1}{N+1}\left(L_{j \alpha}^{\alpha}+\sigma_{j \alpha} \varphi^{\alpha}\right) \quad \text { and } \quad \sigma_{j k}^{i}=-\sigma_{j k} \varphi^{i} .
$$

Therefore, we obtain

$$
\left\{\begin{array}{l}
-\sigma_{j m \mid n}^{i}=\left(\sigma_{j m} \varphi^{i}\right)_{\mid n}=\sigma_{j m \mid n} \varphi^{i}-\sigma_{j m} L_{\alpha n}^{i} \varphi^{\alpha}+\sigma_{j m} v_{n} \varphi^{i}+\sigma_{j m} \mu \delta_{n}^{i} \\
-\sigma_{i j \mid \alpha}^{\alpha}=\left(\sigma_{i j} \varphi^{\alpha}\right)_{\mid \alpha}=\sigma_{i j \mid \alpha} \varphi^{\alpha}-\sigma_{i j} L_{\alpha \beta}^{\beta} \varphi^{\alpha}+\sigma_{i j} v_{\alpha} \varphi^{\alpha}+N \mu \sigma_{i j}, \\
-\sigma_{\alpha i \mid j}^{\alpha}=\left(\sigma_{\alpha i} \varphi^{\alpha}\right)_{\mid j}=\left(\sigma_{i \alpha} \varphi^{\alpha}\right)_{\mid j}= \\
\sigma_{\alpha i \mid j} \varphi^{\alpha}-\sigma_{\beta i} L_{\alpha j}^{\beta} \varphi^{\alpha}+\sigma_{\alpha i} v_{j} \varphi^{\alpha}+\sigma_{1} \sigma_{i j} .
\end{array}\right.
$$

After substituting the expressions $(3.4,3.6,3.7)$ in $\left(2.2^{\prime}, 2.3^{\prime}, 2.9\right)$, we obtain

$$
\begin{aligned}
& \tilde{\mathcal{T}}_{1}^{i}{ }_{j k}=L_{\underline{j k}}^{i}-\frac{1}{N+1} \delta_{k}^{i}\left(L_{\underline{j \alpha}}^{\alpha}+\sigma_{j \alpha} \varphi^{\alpha}\right)-\frac{1}{N+1} \delta_{j}^{i}\left(L_{\underline{k \alpha}}^{\alpha}+\sigma_{k \alpha} \varphi^{\alpha}\right)+\sigma_{j k} \varphi^{i},
\end{aligned}
$$

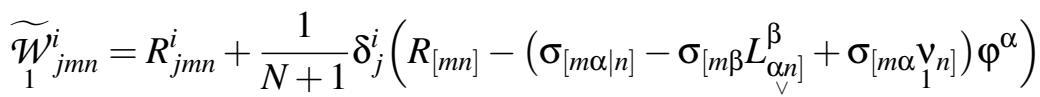

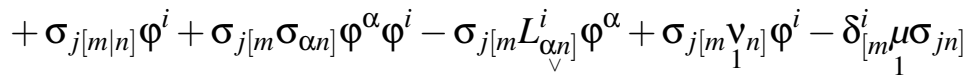

$$
\begin{aligned}
& \left.-\frac{1}{N+1}\left(\delta_{[m}^{i} L_{\underline{j \alpha \mid n]}}^{\alpha}+\left(\delta_{[m}^{i} \sigma_{j \alpha \mid n]}-\delta_{[m}^{i} \sigma_{j \beta} L_{\alpha, n]}^{\beta}+\delta_{[m}^{i} \sigma_{j \alpha} v_{1}\right]\right) \varphi^{\alpha}+\delta_{[m}^{i} \mu \sigma_{j n]}\right) \\
& +\frac{1}{N+1} \delta_{[m}^{i} \sigma_{j n]}\left(L_{\alpha \beta}^{\beta}+\sigma_{\alpha \beta} \varphi^{\beta}\right) \varphi^{\alpha} \\
& -\frac{1}{(N+1)^{2}}\left(L_{\underline{j \alpha}}^{\alpha}+\sigma_{j \alpha} \varphi^{\alpha}\right)\left(\delta_{[m}^{i} L_{n] \beta}^{\beta}+\delta_{[m}^{i} \sigma_{n] \beta} \varphi^{\beta}\right), \\
& \widetilde{W}_{1}^{i}{ }_{j m n}=R_{j m n}^{i}+\frac{1}{N+1} \delta_{j}^{i} R_{[m n]}+\frac{N}{N^{2}-1} \delta_{[m}^{i} R_{j n]}+\frac{1}{N^{2}-1} \delta_{[m}^{i} R_{n] j} \\
& +\sigma_{j[m \mid n]} \varphi^{i}-\sigma_{j[m} L_{\alpha, v]}^{i} \varphi^{\alpha}+\sigma_{j[m} v_{1} v_{n} \varphi^{i}+\sigma_{j[m} \sigma_{\alpha n]} \varphi^{\alpha} \varphi^{i} \\
& \left.-\frac{1}{N+1} \delta_{j}^{i}\left(\sigma_{\alpha[m \mid n]}-\sigma_{\beta[m} L_{\alpha, v]}^{\beta}+\sigma_{\alpha[m} v_{n}\right]\right) \varphi^{\alpha} \\
& +\frac{1}{N-1}\left(\delta_{[m}^{i} \sigma_{j n] \mid \alpha}-\delta_{[m}^{i} \sigma_{j n]} L_{\alpha \beta}^{\beta}+\delta_{[m}^{i} \sigma_{j n]} v_{1}\right) \varphi^{\alpha} \\
& +\frac{1}{N-1}\left(\delta_{[m}^{i} \sigma_{j n]} \sigma_{\alpha \beta}-\delta_{[m}^{i} \sigma_{j \alpha} \sigma_{n] \beta}\right) \varphi^{\alpha} \varphi^{\beta} \\
& \left.-\frac{N}{N^{2}-1}\left(\delta_{[m}^{i} \sigma_{\alpha j \mid n]}-\delta_{[m}^{i} \sigma_{\beta j} L_{\underset{\mathrm{V}}{ }]}^{\beta}+\delta_{[m}^{i} \sigma_{\alpha j} v_{n}\right]\right) \varphi^{\alpha}
\end{aligned}
$$


970

DUŠAN J. SIMJANOVIĆ AND NENAD O. VESIĆ

$$
-\frac{1}{N^{2}-1}\left(\delta_{[m}^{i} \sigma_{\alpha n] \mid j}-\delta_{[m}^{i} \sigma_{\beta n]} L_{\alpha j}^{\beta}+\delta_{[m}^{i} \sigma_{\alpha n]} v_{1} j\right) \varphi^{\alpha} .
$$

Let us express invariant $\widetilde{W}_{1}^{i}$ inn in the form

$$
\begin{aligned}
\widetilde{W}_{1}^{i}{ }_{j m n}= & R_{j m n}^{i}+\frac{1}{N+1} \delta_{j}^{i} R_{[m n]}+\frac{N}{N^{2}-1} \delta_{[m}^{i} R_{j n]}+\frac{1}{N^{2}-1} \delta_{[m}^{i} R_{n] j} \\
& +\delta_{j}^{i} X_{[}[m n] \\
\left.+\delta_{[m}^{i} Y_{1} j n\right] & +Z_{1}^{i}{ }_{j m n},
\end{aligned}
$$

for tensors

$$
\begin{aligned}
X_{i j}= & -\frac{1}{N+1}\left(\sigma_{\alpha i \mid j}-\sigma_{\beta i} L_{\alpha j}^{\beta}+\sigma_{\alpha i} v_{j}\right) \varphi^{\alpha}, \\
Y_{1}= & \frac{1}{N-1}\left(\sigma_{i j \mid \alpha}-\sigma_{i j} L_{\alpha \beta}^{\beta}+\sigma_{i j} v_{\alpha}\right) \varphi^{\alpha} \\
& +\frac{1}{N-1}\left(\sigma_{i j} \sigma_{\alpha \beta}-\sigma_{i \alpha} \sigma_{j \beta}\right) \varphi^{\alpha} \varphi^{\beta} \\
& -\frac{N}{N^{2}-1}\left(\sigma_{\alpha i \mid j}-\sigma_{\beta i} L_{\alpha j}^{\beta}+\sigma_{\alpha i} v_{j}\right) \varphi^{\alpha} \\
& -\frac{1}{N^{2}-1}\left(\sigma_{\alpha j \mid i}-\sigma_{\beta j} L_{\alpha i}^{\beta}+\sigma_{\alpha j} v_{i}\right) \varphi^{\alpha}, \\
Z_{1}^{Z_{j m n}}= & \sigma_{j[m \mid n]} \varphi^{i}-\sigma_{j[m} L_{\alpha n]}^{i} \varphi_{v}^{\alpha}+\sigma_{j[m} v_{n} \varphi^{i}+\sigma_{j[m} \sigma_{\alpha n]} \varphi^{\alpha} \varphi^{i} .
\end{aligned}
$$

After contracting equality $0=\widetilde{\widetilde{W}}_{1}^{i}{ }_{j m n}-\widetilde{W}_{1}^{i}{ }_{j m n}$ by $i$ and $j$, we obtain

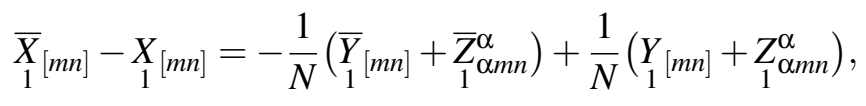

where

$$
\left.Y_{1}[i j]=-\frac{1}{N+1}\left(\sigma_{\alpha[i \mid j]}-\sigma_{\beta[i} L_{\alpha j]}^{\beta}+\sigma_{\alpha[i} v_{j}\right]\right) \varphi^{\alpha} .
$$

After substituting the equations $(3.12,3.13,3.14,3.15)$ in

$$
\begin{aligned}
& 0=\widetilde{W}_{1}^{i}{ }_{j m n}-\widetilde{W}_{1}^{i}{ }_{j m n} \\
& =\bar{R}_{j m n}^{i}-R_{j m n}^{i}+\frac{1}{N+1} \delta_{j}^{i}\left(\bar{R}_{[m n]}-R_{[m n]}\right)+\frac{N}{N^{2}-1}\left(\delta_{[m}^{i} \bar{R}_{j n]}-\delta_{[m}^{i} R_{j n]}\right) \\
& +\frac{1}{N^{2}-1}\left(\delta_{[m}^{i} \bar{R}_{n] j}-\delta_{[m}^{i} R_{n] j}\right)+\delta_{j}^{i}\left(\bar{X}_{[m n]}-X_{1}[m n]\right) \\
& +\left(\delta_{[m}^{i} \bar{Y}_{1}{ }_{j n]}-\delta_{[m}^{i} Y_{j n]}\right)+\bar{Z}_{1}^{i}{ }_{j m n}-Z_{1}^{i}{ }_{j m n},
\end{aligned}
$$




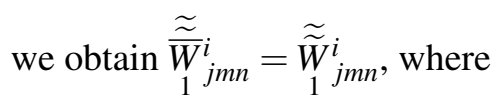

$$
\begin{aligned}
\widetilde{\widetilde{W}}_{1}^{i}{ }_{j m n}= & R_{j m n}^{i}+\frac{1}{N+1} \delta_{j}^{i} R_{[m n]}+\frac{N}{N^{2}-1} \delta_{[m}^{i} R_{j n]}+\frac{1}{N^{2}-1} \delta_{[m}^{i} R_{n] j} \\
& +\sigma_{j[m \mid n]} \varphi^{i}-\sigma_{j[m} L_{\alpha, v]}^{i} \varphi^{\alpha}+\sigma_{j[m} v_{n]} \varphi^{i}+\sigma_{j[m} \sigma_{\alpha n]} \varphi^{\alpha} \varphi^{i} \\
& -\frac{N}{N+1} \delta_{j}^{i}\left(\sigma_{\alpha[m \mid n]}-\sigma_{\beta[m} L_{\alpha n]}^{\beta}+\sigma_{\alpha[m} v_{1}\right) \varphi^{\alpha} \\
& +\frac{1}{N-1}\left(\delta_{[m}^{i} \sigma_{j n] \mid \alpha}-\delta_{[m}^{i} \sigma_{j n]} L_{\alpha \beta}^{\beta}+\delta_{[m}^{i} \sigma_{j n]} v_{1}\right) \varphi^{\alpha} \\
& +\frac{1}{N-1}\left(\delta_{[m}^{i} \sigma_{j n]} \sigma_{\alpha \beta}-\delta_{[m}^{i} \sigma_{j \alpha} \sigma_{n] \beta}\right) \varphi^{\alpha} \varphi^{\beta} \\
& -\frac{N}{N^{2}-1}\left(\delta_{[m}^{i} \sigma_{\alpha j \mid n]}-\delta_{[m}^{i} \sigma_{\beta j} L_{\alpha n]}^{\beta}+\delta_{[m}^{i} v_{n]}\right) \varphi^{\alpha} \\
& -\frac{1}{N^{2}-1}\left(\delta_{[m}^{i} \sigma_{\alpha n] \mid j}-\delta_{[m}^{i} \sigma_{\beta n]} L_{\alpha j}^{\beta}+\delta_{[m}^{i} \sigma_{\alpha n]} v_{j}\right) \varphi^{\alpha} .
\end{aligned}
$$

Based on invariants $\widetilde{W}_{1}^{i}{ }_{j m n}$ and $\widetilde{\widetilde{W}}_{1}^{i}{ }_{j m n}$, we can conclude that geometrical object $\left.\left(\sigma_{\alpha[i \mid j]}-\sigma_{\beta[i} L_{\alpha j]}^{\beta}+\sigma_{\alpha[i} v_{j}\right]\right) \varphi^{\alpha}$ is an invariant for mapping $f$. For this reason, invariant $\widetilde{W}_{1}^{i}{ }_{j m n}$ given by (3.10) reduces to

$$
\begin{aligned}
\widetilde{W}_{1 j m n}^{i}= & R_{j m n}^{i}+\frac{1}{N+1} \delta_{j}^{i} R_{[m n]}+\frac{N}{N^{2}-1} \delta_{[m}^{i} R_{j n]}+\frac{1}{N^{2}-1} \delta_{[m}^{i} R_{n] j} \\
& +\sigma_{j[m \mid n]} \varphi^{i}-\sigma_{j[m} L_{\alpha n]}^{i} \varphi^{\alpha}+\sigma_{j[m} v_{n} \varphi^{i}+\sigma_{j[m} \sigma_{\alpha n]} \varphi^{\alpha} \varphi^{i} \\
& +\frac{1}{N-1}\left(\delta_{[m}^{i} \sigma_{j n] \mid \alpha}-\delta_{[m}^{i} \sigma_{j n]} L_{\alpha \beta}^{\beta}+\delta_{[m}^{i} \sigma_{j n]} v_{\alpha}\right) \varphi^{\alpha} \\
& +\frac{1}{N-1}\left(\delta_{[m}^{i} \sigma_{j n]} \sigma_{\alpha \beta}-\delta_{[m}^{i} \sigma_{j \alpha} \sigma_{n] \beta}\right) \varphi^{\alpha} \varphi^{\beta} \\
& -\frac{N}{N^{2}-1}\left(\delta_{[m}^{i} \sigma_{\alpha j \mid n]}-\delta_{[m}^{i} \sigma_{\beta j} L_{\alpha n]}^{\beta}+\delta_{[m}^{i} v_{n]}\right) \varphi^{\alpha} \\
& -\frac{1}{N^{2}-1}\left(\delta_{[m}^{i} \sigma_{\alpha n] \mid j}-\delta_{[m}^{i} \sigma_{\beta n]} L_{\alpha j}^{\beta}+\delta_{[m}^{i} \sigma_{\alpha n]} \nu_{j}\right) \varphi^{\alpha} .
\end{aligned}
$$

If contract equality $0=\widetilde{W}_{1}^{i}{ }_{j m n}-\widetilde{W}_{1}^{i}{ }_{j m n}$ (equivalent to the equation (3.16)) by $i$ and $n$ and anti-symmetrize the contracted equation by $j$ and $m$, we will obtain

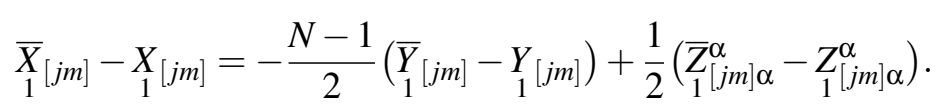




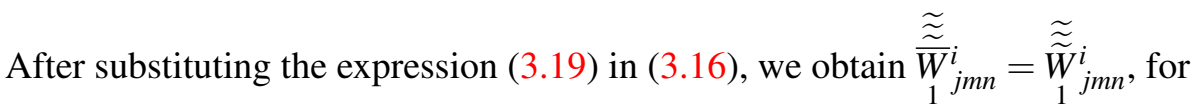

$$
\begin{aligned}
& {\underset{\widetilde{W}}{j}}_{j m n}^{i}=R_{j m n}^{i}+\frac{1}{N+1} \delta_{j}^{i} R_{[m n]}+\frac{N}{N^{2}-1} \delta_{[m}^{i} R_{j n]}+\frac{1}{N^{2}-1} \delta_{[m}^{i} R_{n] j} \\
& +\sigma_{j[m \mid n]} \varphi^{i}-\sigma_{j[m} L_{\alpha n]}^{i} \varphi^{\alpha}+\sigma_{j[m} \nu_{n]} \varphi^{i}+\sigma_{j[m} \sigma_{\alpha n]} \varphi^{\alpha} \varphi^{i}
\end{aligned}
$$

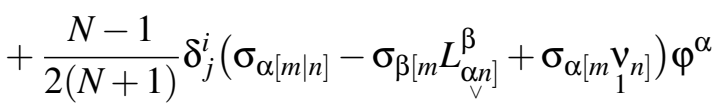

$$
\begin{aligned}
& -\frac{1}{2} \delta_{j}^{i}\left(\sigma_{[m \alpha \mid n]}-\sigma_{[m \beta} L_{\alpha, n]}^{\beta}+\sigma_{[m \alpha} v_{1}\right) \varphi^{\alpha} \\
& +\frac{1}{N-1}\left(\delta_{[m}^{i} \sigma_{j n] \mid \alpha}-\delta_{[m}^{i} \sigma_{j n]} L_{\alpha \beta}^{\beta}+\delta_{[m}^{i} \sigma_{j n]} v_{\alpha}\right) \varphi^{\alpha} \\
& +\frac{1}{N-1}\left(\delta_{[m}^{i} \sigma_{j n]} \sigma_{\alpha \beta}-\delta_{[m}^{i} \sigma_{j \alpha} \sigma_{n] \beta}\right) \varphi^{\alpha} \varphi^{\beta} \\
& -\frac{N}{N^{2}-1}\left(\delta_{[m}^{i} \sigma_{\alpha j \mid n]}-\delta_{[m}^{i} \sigma_{\beta j} L_{\alpha, v]}^{\beta}+\delta_{[m}^{i} \sigma_{\alpha j} v_{n]}\right) \varphi^{\alpha} \\
& -\frac{1}{N^{2}-1}\left(\delta_{[m}^{i} \sigma_{\alpha n] \mid j}-\delta_{[m}^{i} \sigma_{\beta n]} L_{\alpha j}^{\beta}+\delta_{[m}^{i} \sigma_{\alpha n]}{ }_{1}{ }_{j}\right) \varphi^{\alpha} \text {. }
\end{aligned}
$$

After comparing invariants $\underset{1}{\underset{\widetilde{W}}{i}}{ }_{j m n}^{i}$ and $\widetilde{W}_{1}^{i}$ jmn for mapping $f$, we obtain that invariant $\widetilde{\widetilde{W}}_{1}^{i}{ }_{j m n}$ given by (3.20) reduces to invariant $\widetilde{W}_{1}^{i}{ }_{j m n}$ given by (3.18).

Based on the transformation of family (1.7) of curvature tensors of space $\mathbb{G A}_{N}$ under mapping $f$, we obtain:

$$
\begin{aligned}
& \mathcal{W}_{1}^{i}{ }_{0 .\left(p^{1}\right) \cdot\left(p^{2}\right) \cdot j m n}=\widetilde{\mathcal{W}}_{1}^{i}{ }_{j m n}+u L_{j \mathrm{v} \mid n}^{i}+u^{\prime} L_{j n \mid m}^{i} \\
& -u\left(\omega_{\left(p_{1}^{1}\right) . \alpha n}^{i} L_{j m}^{\alpha}-\omega_{\left(p_{2}^{1}\right) . j n}^{\alpha} L_{\alpha,}^{i}-\omega_{\left(p_{3}^{1}\right) . m n}^{\alpha} L_{j \alpha}^{i}\right) \\
& -u^{\prime}\left(\omega_{\left(p_{1}^{2}\right) \cdot \alpha m}^{i} L_{j n}^{\alpha}-\omega_{\left(p_{2}^{2}\right) . j m}^{\alpha} L_{\vee}^{i}-\omega_{\left(p_{3}^{2}\right) . m n}^{\alpha} L_{j \alpha}^{i}\right) \text {, } \\
& W_{1}^{i}{ }_{0 .\left(p^{1}\right) \cdot\left(p^{2}\right) \cdot j m n}=\widetilde{W}_{1}^{i}{ }_{j m n}+u L_{j m \mid n}^{i}+u^{\prime} L_{j n \mid m}^{i} \\
& -u\left(\omega_{\left(p_{1}^{1}\right) \cdot \alpha n}^{i} L_{j m}^{\alpha}-\omega_{\left(p_{2}^{1}\right) \cdot j n}^{\alpha} L_{\vee}^{i}-\omega_{\left(p_{3}^{1}\right) \cdot m n}^{\alpha} L_{\vee v}^{i}\right) \\
& -u^{\prime}\left(\omega_{\left(p_{1}^{2}\right) . \alpha m}^{i} L_{\vee j}^{\alpha}-\omega_{\left(p_{2}^{2}\right) . j m}^{\alpha} L_{\vee}^{i}-\omega_{\left(p_{3}^{2}\right) . m n}^{\alpha} L_{j \alpha}^{i}\right),
\end{aligned}
$$

for $p_{1}^{1}, \ldots, p_{3}^{2} \in\{1,2\}, \omega_{(1) . j k}^{i}=L_{\underline{j k}}^{i}$ and $\omega_{(2) . j k}^{i}=\omega_{j k}^{i}$, for geometrical object $\omega_{j k}^{i}$ given in (3.4).

The next theorem holds. 
Theorem 1. Let $f: \mathbb{G A}_{N} \rightarrow \mathbb{G}_{N}$ be an equitorsion almost geodesic mapping of the type $\pi_{3}$.

The geometrical object $\tilde{\mathcal{T}}^{i}{ }_{j k}$ given by (3.8) is basic invariant of Thomas type for mapping $f$. The invariance of this geometrical object is total.

Geometrical object $\widetilde{\mathcal{W}}_{\text {jmn }}^{i}$ given by (3.9) is basic associated invariant of Weyl type for mapping $f$. The invariance of this geometrical object is valued. It is total if and only if mapping $f$ has the property of reciprocity.

The geometrical object $\widetilde{W}_{j m n}^{i}$ given by (3.18) is associated derived invariant of the Weyl type for mapping $f$. The invariance of this geometrical object is valued. It is total if and only if the mapping $f$ has the property of reciprocity.

Geometrical objects ${\underset{1}{W}}_{j m n}^{i}, \underset{1}{W_{j m n}^{i}}$, given by $(3.21,3.22)$, are invariants for the equitorsion third type almost geodesic mapping $f$. The invariance of these geometrical objects is valued. This invariance is total if and only if mapping $f$ has the property of reciprocity.

\section{CONClusion}

We have obtained new invariants for the almost geodesic mappings of the third type of a non-symmetric affine connection space in this paper.

In Section 3, the invariants for equitorsion almost geodesic mappings of the third type are presented. The method used for obtaining these invariants (see [20]), simplified the corresponding method presented by Sinyukov [14] and used latter in [19,21].

The results obtained in this paper motivate the authors to continue their research about invariants for almost geodesic mappings of non-symmetric affine connection spaces.

\section{ACKNOWLEDGEMENT}

The authors thank to the anonymous referee who estimated the quality of this paper.

\section{REFERENCES}

[1] O. Belova, J. Mikeš, and K. Strambach, “About almost geodesic curves.” Filomat, vol. 33, no. 4, pp. 1013-1018, 2019, doi: 10.2298/FIL1904013B.

[2] V. Berezovski and J. Mikeš, “Almost geodesic mappings of spaces with affine connection.” $J$. Math. Sci., vol. 207, no. 3, pp. 389-409, 2015, doi: 10.1007/s10958-015-2378-5.

[3] V. Berezovski, J. Mikeš, L. Rýparová, and A. Sabykanov, "On canonical almost geodesic mappings of type $\pi_{2}(e) . "$, vol. 54, no. 8, 2020, doi: 10.3390/math8010054.

[4] L. P. Eisenhart, Non-Riemannian geometry. New York: American Mathematical Society (AMS), Providence, RI, 1927. doi: 10.1002/zamm.19290090633.

[5] J. Mikeš and S. Báscó et al., Differential geometry of special mappings, 2nd edition. Olomouc: Palacký University, Faculty of Science, 2019. doi: 10.5507/prf.19.24455358. 
[6] S. M. Minčić, "Curvature tensors of the space of non-symmetric affine connexion, obtained from the curvature pseudotensors." Mat. Vesn., N. Ser., vol. 13, no. 28, pp. 421-435, 1976.

[7] S. M. Minčić, "Independent curvature tensors and pseudotensors of spaces with non-symmetric affine connexion.” Coll. Math. Soc. János Bolayai, Dif. geom., vol. 31, pp. 445-460, 1979.

[8] S. M. Minčić, "On Ricci type identities in manifolds with non-symmetric affine connection." Publ. Inst. Math., Nouv. Sér., vol. 94, no. 108, pp. 205-217, 2013, doi: 10.2298/PIM1308205M.

[9] S. M. Minčić and M. S. Stanković, "Equitorsion geodesic mappings of generalized Rimannian spaces." Publ. de l'Institut Math. Nouv. Sér., vol. 61, no. 75, pp. 97-104, 1997.

[10] S. M. Minčić and L. S. Velimirović, "Spaces with non-symmetric affine connection." Novi Sad J. Math., vol. 38, no. 3, pp. 157-164, 2008.

[11] D. Mušicki and B. Milić, Mathematical foundations of theoretical physics with a collection of solved problems (in Serbian). Bulevar Vojvode Mišića 17: University of Belgrade, 1975.

[12] M. Z. Petrović, "Canonical almost geodesic mappings of type $\pi_{2}(0, F), \theta \in\{1,2\}$, between generalized parabolic Kähler manifolds.” Miskolc Math. Notes, vol. 19, no. 1, pp. 469-482, 2018, doi: 10.18514/MMN.2018.1908.

[13] M. Z. Petrović and M. S. Stanković, "Special almost geodesic mappings of the first type of nonsymmetric affine connection spaces.” Bull. Malays. Math. Sci. Soc., vol. 40, no. 3, pp. 1353-1362, 2017, doi: 10.1007/s40840-015-0118-0.

[14] N. S. Sinyukov, Geodesic mappings of Riemannian spaces. Moscow: Nauka, (in Russian), 1979.

[15] V. S. Sobchuk, J. Mikeš, and O. Pokorná, "On almost geodesic mappings $\pi_{2}$ between semisymmetric Riemannian spaces.” Novi Sad J. Math., vol. 29, no. 3, pp. 309-312, 1999.

[16] M. S. Stanković, "First type almost geodesic mappings of general affine connection spaces." Novi Sad J. Math., vol. 29, no. 3, pp. 313-323, 1999.

[17] M. S. Stanković, "On a canonic almost geodesic mappings of the second type of affine spaces." Filomat, vol. 13, pp. 105-114, 1999.

[18] M. S. Stanković, "On a special almost geodesic mapping of third type of affine spaces." Novi Sad J. Math., vol. 31, no. 2, pp. 125-135, 2001.

[19] M. S. Stanković, "Special equitorsion almost geodesic mappings of the third type of nonsymmetric affine connection spaces." APPL. MATH. COMPUT., vol. 244, pp. 695-701, 2014, doi: 10.1016/j.amc.2014.07.021.

[20] N. O. Vesić, "Basic invariants of geometric mappings." Miskolc Math. Notes, vol. 21, no. 1, pp. 473-487, 2020, doi: 10.18514/MMN.2020.2901.

[21] N. O. Vesić, L. S. Velimirović, and M. S. Stanković, "Some invariants of equitorsion third type almost geodesic mappings." Mediterr. J. Math., vol. 13, no. 6, pp. 4581-4590, 2016, doi: 10.1007/s00009-016-0763-z.

[22] M. L. Zlatanović, "New projective tensors for equitorsion geodesic mappings." Appl. Math. Lett., vol. 25, no. 5, pp. 890-897, 2011, doi: 10.1016/j.aml.2011.10.045.

[23] M. L. Zlatanović, "On equitorsion geodesic mappings of general affine connection spaces onto generalized Riemannian spaces.” Appl. Math. Lett., vol. 24, no. 5, pp. 665-671, 2011, doi: 10.1016/j.aml.2010.12.003.

\section{Authors' addresses}

Dušan J. Simjanović

(Corresponding author) Metropolitan University, Tadeuša Košćuška 63, 11158 Belgrade, Serbia

Current address: Faculty of Information Technology, Bulevar Sv. Cara Konstantina 80A, 18116 Niš, Serbia,

E-mail address: dsimce@gmail.com 
Nenad O. Vesić

Mathematical Institute, Serbian Academy of Sciences and Arts, Kneza Mihaila 35, 11000 Belgrade, Serbia

E-mail address: n.०.vesic@out look.com 